\title{
PACKET ROUTING - PACKET TRAVELS ON NETWORK FROM SOURCE TO DESTINATION - COMPLEXITY \& ALGORITHMS
}

\author{
R. Idayathulla ${ }^{1}$, Dr. M. Robinson Joel ${ }^{2}$ \\ ${ }^{1}$ Research Scholar, Department of Computer Science, Ponnaiyah Ramajayam Institute \\ of Science and Technology (Deemed to be University), Thanjavur, Tamil Nādu, India \\ ${ }^{2}$ Associate Professor, Department of Information Technology, Kings Engineering \\ College, Chennai, Tamil Nādu, India \\ ${ }^{1 *}$ Corresponding Author Email: idayathulla86@gmail.com
}

\section{ABSTRACT:}

Bundle steering is one of the fundamental issues in PC networks wherein a switch decides the following bounce of each parcel with inside the line to get it as quick as reasonable to its objective. Every switch utilizes an inward directing work area to choose the quality course to deliver a parcel. A steering work area is a fixed of rules, consistently thought to be in work area design, this is utilized to choose wherein realities bundles visiting over an Internet Protocol (IP) people group could be coordinated.

Store-and-ahead bundle steering has a place with the most extreme fundamental commitments in local area advancement. Restricted data transmission requires that a couple of bundles can't course to their place for getting away on the double anyway need to go to at halfway hubs on their bearing or take diversions. Specifically, for time pivotal applications, it's far ideal to find plans that make specific rapid delivery of the bundles. It is along these lines a home-grown objective to decrease the make length, i.e., the time at which a definitive parcel shows up at its place for getting away. In this paper we gift various ground-breaking considerations and procedures that cause novel calculations and hardness results.

In this paper we take a gander at the parcel directing problem. Given a fixed of bundles locally beginning at likely remarkable start vertices, we want to move them to their separate place to get-away vertices. The reason for existing is to diminish the general make length, this is the time while the last bundle shows up at its place for getting away. We recall the disconnected model of the issue wherein all information around the local area and the parcels, predominantly the start and place for getting away vertices, are given prior. In our directing model, we expect shop-and-ahead steering. This way that every hub can shop discretionarily numerous parcels anyway every connection (a coordinated or undirected edge) might be used by least complex each bundle in turn. We check out the case wherein the path of the bundles is steady prior notwithstanding the case wherein their calculation is a piece of the problem. Also, we recognize among remarkable assortments of hidden diagrams, e.g., coordinated charts, undirected charts, planar charts or trees [1].

Keywords: Data Packets, Routing Techniques, Internet Protocol Address, Bellman -Ford Algorithms, Dynamic Programming. 


\section{INTRODUCTION}

I'm diverging from my subjects' calculations and running gadget because of the reality this question is presently a day is typically mentioned in interviews. All the more so in any systems administration related associations interviews like CISCO, Juniper, ALU and QUALCOMM. Question is the means by which does a parcel goes from supply to place for getting away in web. This is to check on the off chance that your secure bundle steering. There are 3 components to the question. First is the thing that happens in the pc or host while a parcel is produced with the guide of utilizing application. Second part is the manner in which it goes from one host this is supply to various host this is place to get-away with numerous switches sitting in the middle. Third part is the thing that is going inside place for getting away host while it gets a bundle on network. We will talk each one with the guide of utilizing one [2].

Steering is the strategy for finding a heading among hubs locally essentially dependent on certain guidelines which incorporate the briefest course or the fastest bearing and so forth Current steering calculations Endeavor to find the top-notch course among the stockpile and objective [3].

To change a bundle from supply to place for getting away, each the MAC adapt to and IP adapt to of the place for getting away should be known. Assuming the place for getting away MAC adapt to isn't gift, then, at that point, ARP will cure this issue first then the bundle might be brought to a place for getting away host. There are simple rules for a bundle drift in an organization:

In case the place to get-away host is gift withinside the equivalent local area in light of the fact that the stock host then the bundle may be added immediately to the place for getting away host the utilization of MAC manage. Inside a local area, the parcel may be added on the reason of MAC manage. Macintosh manage in no way, shape or form crosses its transmission area [4].

Static methodology is the just wherein the pc overseer physically units the IP manage to the gadget. In case your gadget is appended to a local area like LAN then one issue is to be put away in considerations that the IP manage being set should now presently don't be like the IP manage of one more gadget at the indistinguishable local area as this could bring about IP manage fighting and none of the 2 machines may be fit for get right of passage to the web. Dynamic methodology is the just wherein the pc (on gadget boot) requests that a server appoint an IP manage to it. The convention utilized for this method is alluded to as Dynamic Host Control Protocol (DHCP). The server referred to here is perceived DHCP server. This waiter is responsible for doling out IP locations to all of the PC frameworks at the local area. It is the obligation of the DHCP server to guarantee that there might be no IP manage fighting. Assuming one of the gadgets is going down and on the other hand boots up then a shimmering DHCP demand is despatched to the server which may likewise dole out the indistinguishable or a couple of particular IPS manage this time. Normally, a pool of IP addresses is given to the DHCP server and it utilizes least complex the ones IP addresses for tasks. This is accomplished to sufficiently utilize distinctive IP addresses for static tasks with none fighting. 


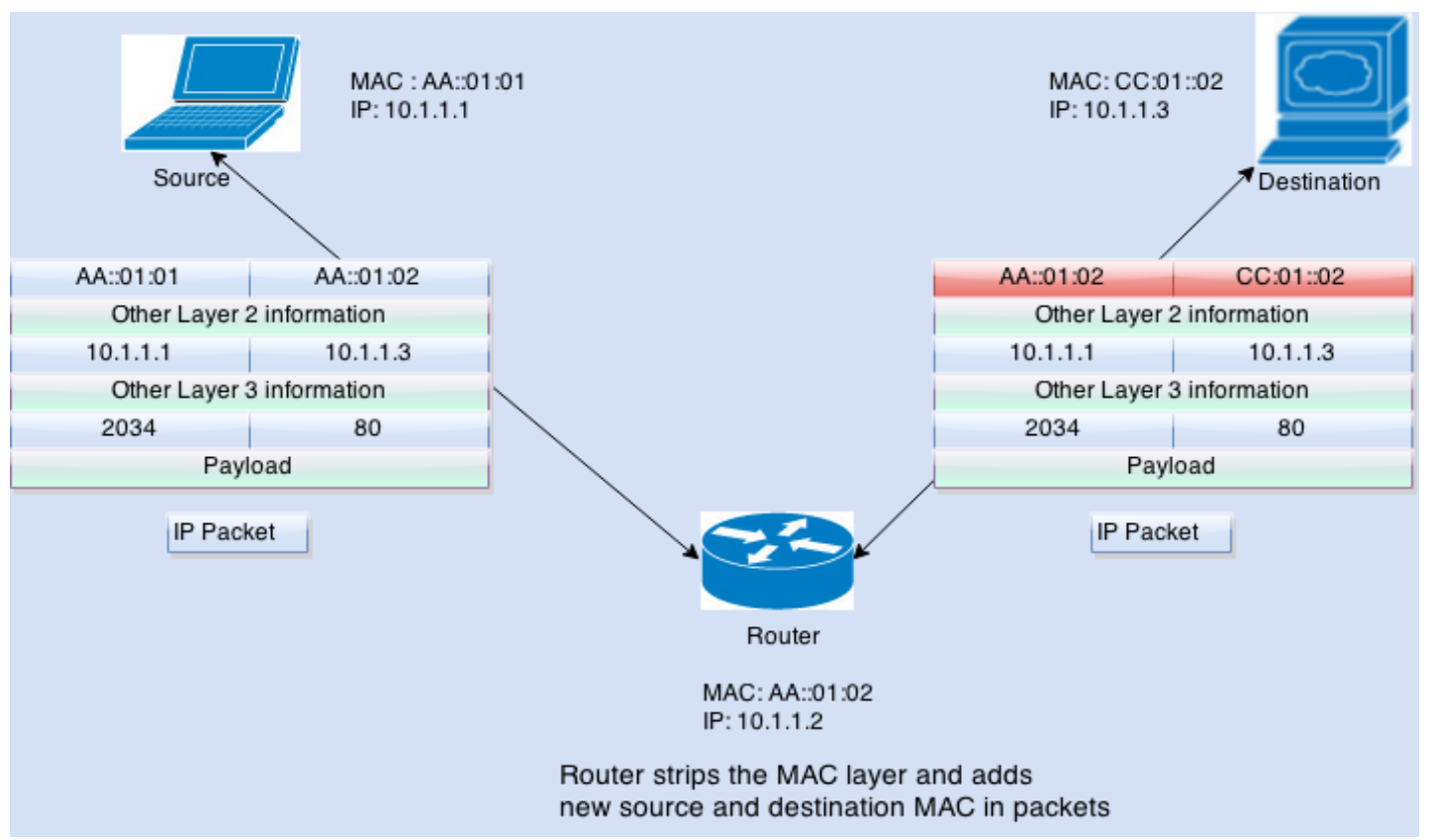

Figure 1: Router Strips from Source to Destination

The space of guest's undertaking calculations has been a fiery area of studies for more than fifty years and by and by remains so for 2 basic reasons. In the first place, it decides the expected guests float test and recognizes the scopes of supplier withinside the transportation organization, and desk work the reason for evaluating the charges and benefits of the capacity choices for

Transportation mediation projects. Second, the arrangement of consistently better computational abilities and the need to adapt to answer assembly inconveniences from a professional point has propelled studies to upgrade the general exhibition of site guests project calculations. The individual harmony principally based absolutely static site guests project (UESTA) (Wardrop, 1952) is a generally standard methodology for long-lasting period arranging. Supported examination on this area have achieved many site guests project calculations to cure the UESTA issue, starting with the Frank-Wolfe (F-W) calculation (Frank and Wolfe, 1956) that is popular in practice as a result of its straightforwardness of execution [5].

Way basically based absolutely calculations (PBAs) comprising of the disaggregate simplicial deterioration set of rules (Larson and Patriksson, 1992), the slope projection set of rules (Jayakrishnan et al., 1994), and the projected inclination set of rules (Florian et al., 2009), notwithstanding the bushbased calculations (BBAs) comprising of the beginning essentially based completely set of rules (Bar-Gera, 2002) and the arrangement of rules B (Dial, 2006), were demonstrated to do faster in accomplishing intermingling when contrasted with hyperlink-principally based absolutely calculations containing the F-W set of rules (Lee et al., 2002; Florian et al., 2009). Further,

for finding the responses for scarcely restrictive data sources the utilization of a hotness starts, that is explicitly helpful in examples wherein guests task wishes to be performed: (1) for assessing the more than one choice of local area enhancements which range from the base case 
with the guide of utilizing around certain connections, and (2) for scarcely selective experience requests (Peeta et al., 2011). Further, hyperlink streams might be procured in a true manner with the guide of utilizing totalling the course streams, but the convey isn't accurate. Likewise, even as many making arrangements programs require hyperlink streams no one but, others can likewise require course streams. Thus, PBAs and BBAs can give goliath cost when contrasted with their hyperlink-fundamentally based absolutely partners. This article works in PBAs, but the experiences from this pamphlet additionally can be utilized for BBAs [6].

\section{THE ANATOMY OF A PACKET PATH (ICMP) FROM SOURCE TO DESTINATION}

Two of the greatest not unusual place obligations which can be completed with the guide of utilizing a local area engineer are the utilization of the ping and traceroute (tracert) utilities. This article takes a concentrate how the IP bundle is based and steered after which sentiments how each the ping and traceroute (tracert) utilities use IP/ICMP parcels to complete their capacities.

Two of the greatest not unusual place obligations which are done through method of method for a local area engineer are the use of ping and the utilization of traceroute (tracert). A talented specialist is equipped for utilize those guidelines to acquire an aptitude of some of elite issues; those capabilities especially come from a skill of the manner in which the IP parcel is set up and the manner in which guests is directed. This article takes a concentrate how the IP parcel is set up and steered, after which studies how each the ping and traceroute (tracert) utilities use IP/ICMP bundles to do their capacities.

\section{IP PACKET AND IP PACKET ROUTING}

Before the assessment of a bundle course can really start, a couple of amounts of data in how an IP

\begin{tabular}{|c|c|c|c|c|}
\hline Version & $\mathbb{H H}$ & TOS & \multicolumn{2}{|r|}{ Length } \\
\hline \multicolumn{3}{|c|}{ Identification } & Flags & Fragment Offset \\
\hline & & Protoco & \multicolumn{2}{|r|}{ Chedksum } \\
\hline \multicolumn{5}{|c|}{ Souroe Address } \\
\hline \multicolumn{5}{|c|}{ Destination Address } \\
\hline \multicolumn{4}{|c|}{ Options } & Padding \\
\hline
\end{tabular}

Figure 2: IPv4 Header

At the point when the utilization of the ping programming, the architect wants to have a crucial idea of the manner in which a bundle is directed from an inventory to place to get-away; on the grounds that the data of IP steering are contained inside many pages of various books, this pamphlet will best cowl the key idea of IP steering. At the point when an IP parcel is despatched from a stock to place for getting away there are a few stages which may be taken to conclude how definitively the sending devices treats the bundle. Assuming the parcel is 
bound for some other IP manage withinside the equivalent subnet, the bundle will truly be despatched the utilization of Layer 2 procedures (i.e., ARP). Assuming that the bundle is bound to a different subnet, the instrument will assessment its present steering work area for a specific recognized course to the alleged place for getting away. Assuming this bearing exists, the bundle is despatched close by this recognized course; in the event that a specific heading isn't recognized, a default heading is utilized, in case it exists; on the off chance that neither of those exist, the parcel is dropped and a mix-up given. (What and the manner in which the ping programming works is covered in more prominent component withinside the What is a Ping area.) The traceroute programming moreover requires a skill of IP directing anyway also comprehension of the way the Time to Live (TTL) subject is utilized withinside the IP parcel.

\section{PROCESSING PACKET AT SOURCE MACHINE}

Application produces a bundle to be despatched at the local area and boat it to layer beneath. The resulting layer is referred to as conveyance layer which oversees surrender to surrender discussion among machines. The convention utilized might be TCP or UDP. What is qualification among those convention is some other concern by and large. Whenever bundle is formed at conveyance layer, it's far despatched to local area layer which gives supply and place to get-away IP withinside the parcel. Most basic subject that is presented at IP or local area layer is Time to Live (TTL) that is used by middle of the road switches/changes to decide whether bundle wants to be sent or not. (How place to get-away IP is found?) After people group layer, bundle arrives at records hyperlink or MAC layer, in which supply and place for getting away MAC manage of machines are presented. We will perceive how those fields substitute among each neighbour. (How place to get-away MAC is found?) Data hyperlink layer push this parcel to real layer wherein it's far despatched as course of " 0 " and "1" on substantial medium accessible. Parcel more prominent issues are being done at each layer like MTU decision at conveyance, discontinuity at IP and records hyperlink layer and so forth, but for straightforwardness of clarification, I really have skipped them. Presently bundle has reached at a middle switch which sit down among supply and place for getting away like demonstrated in figure [6].

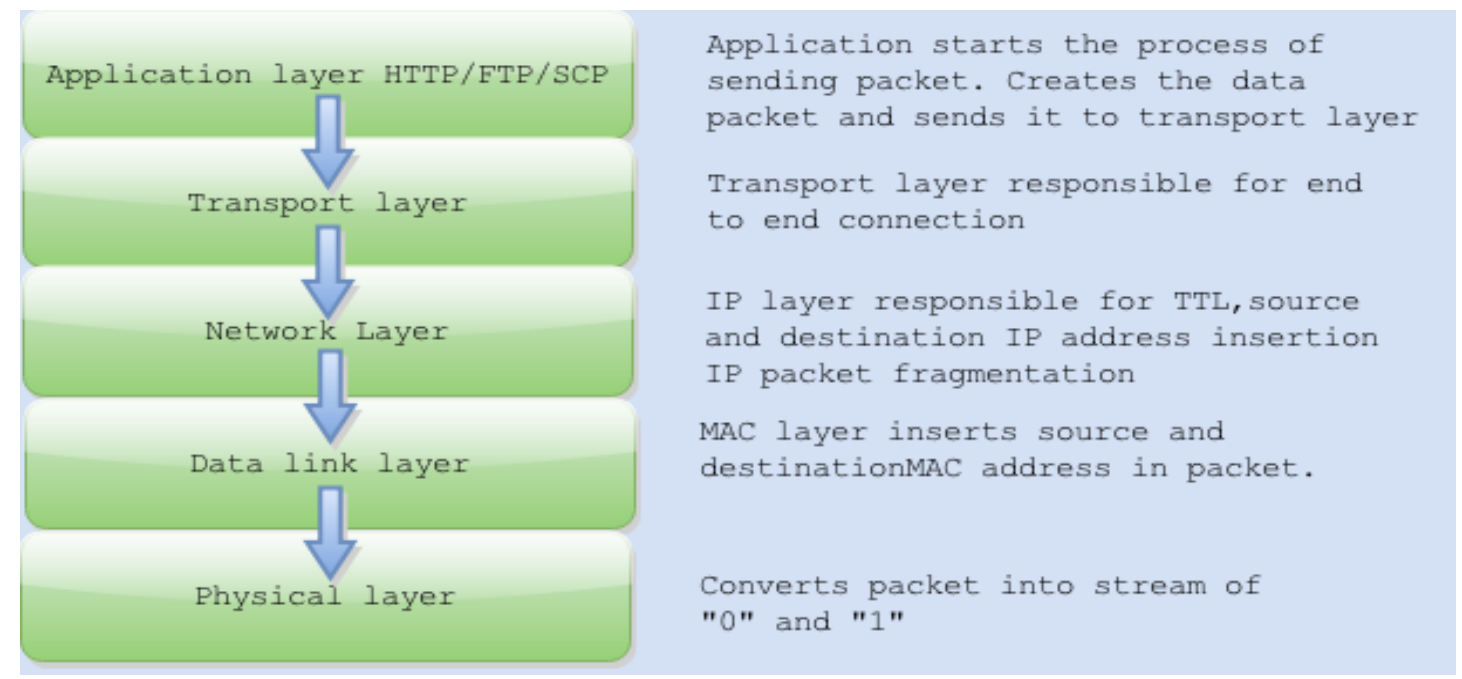

Figure 3: Packet transfer from Source to Destination 
I really have skipped them. Presently bundle has reached at a middle switch which sit down among supply and place for getting away like demonstrated in figure [6].

\section{UNDERSTANDING THE NEED TO "ROUTING ALGORITHMS" [10]}

Locally wherein loads of bundles venture among Source and place for getting away. It is exceptionally basic how tons esteem a parcel takes to travel from supply to place to getaway. Thus, a local area which utilizes substantially less worth to impart records from supply to place to get-away might be alluded to as a green local area. Along these lines, to achieve this,

we need green steering calculations which might be utilized to find the way wherein the bundle mush venture all together that it will accomplish the place to get-away with extremely low worth usage. [10]

Distinguishing the measurements that can be utilized in choosing the "Directing calculations" [10]

The basic measurements which might be utilized to choose a steering calculation are:

- Time intricacy of the calculation

- Space intricacy of the calculation

- Exact

- Consistency

\section{6. "BELLMAN-FORD ALGORITHM" [11]}

Bellman-Ford set of rules is utilized to the most limited course from a solitary inventory diverse vertices in a chart. It furthermore identifying helpless weight cycles, which impediment of Dijkstra 's set of rules. portage set of rules takes settled for its time intricacy is $\mathrm{O}(\mathrm{n} 2)$. Dynamic Programming in which we the cost when and save it in a grid and use them while there 's process the equivalent value we simply the network or cluster. Contrasted with Ford, Dijkstra is higher in wording intricacy as it runs in $\mathrm{O}($ nlogn) the worry line approach (Greed Algorithm). In any case, the standard addition bellman-portage is sorting out poor in this calculation in each progression we save the edges, so we endeavor out all reasonable pick out the charming one, that's how programming calculations work. A few directors used in Bellman-Ford Algorithm - All edges expense might be every helpless positive. - The chart might be coordinated or undirected - The diagram should be associated. Examination and Engineering Development- - Volume four Issue Available at www.ijsred.com CIJSRED: All Rights are Reserved to figure supply to all also permits in that is the arrangement of rules. Bellman for circles so This uses we register an exhibit or a need to utilize it from to Bellman terms of time $\mathrm{O}(\mathrm{nlogn})$ utilizing approach (Greed gain of helpless edges. protect loosening up suitable techniques and how unique Some administrators used in Bellman-Ford Algorithm.

- All edges worth might be each bad sure.

- The chart might be coordinated or undirected

- The chart should be associated 


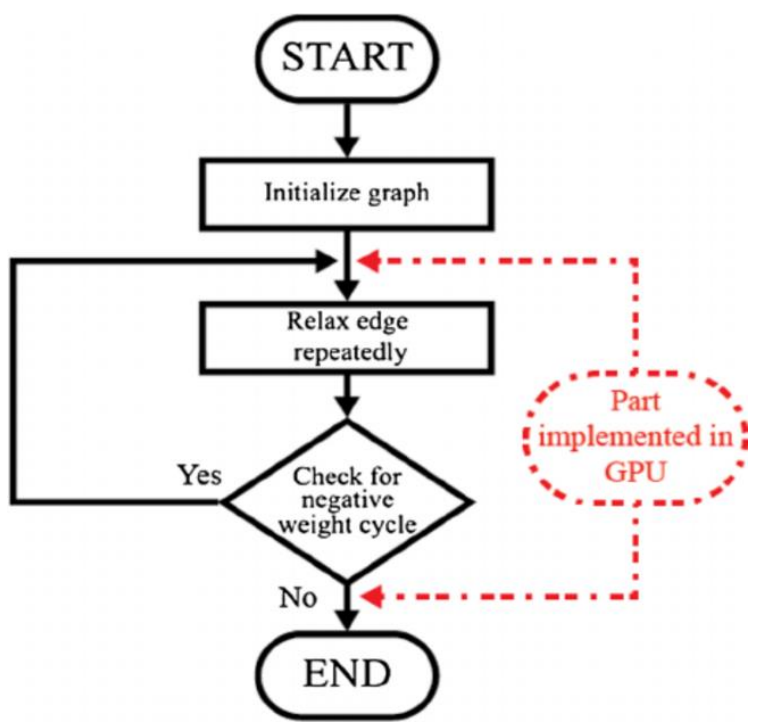

Figure 4: Flow Diagram of Bellman - Ford Algorithm

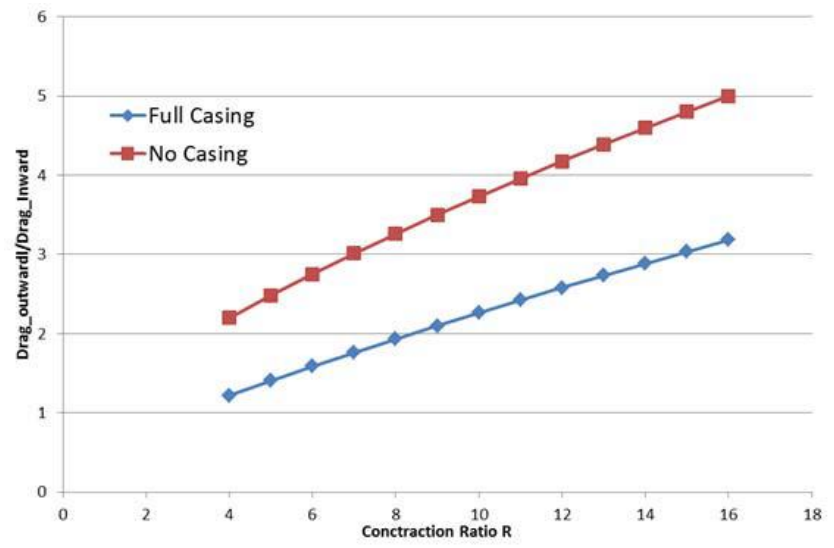

Figure 5: Packet Data Transfer from Source to

Destination

TABLE 1. COMPARING DIFFERENT ALGORITHMS

\begin{tabular}{l|l|l|l|l|} 
Metrics & Dijkstra & $\begin{array}{l}\text { Bellman- } \\
\text { Ford }\end{array}$ & $\begin{array}{l}\text { Floyd } \\
\text { Warshall }\end{array}$ & Johnson \\
\hline $\begin{array}{l}\text { Time } \\
\text { Complexity }\end{array}$ & $\begin{array}{l}\mathrm{O}(\mathrm{E}+ \\
\mathrm{VlogV})\end{array}$ & $\mathrm{O}(\mathrm{VE})$ & $\mathrm{O}\left(\mathrm{V}^{3}\right)$ & $\begin{array}{l}\mathrm{O}\left(\mathrm{V}^{4} \log \mathrm{V}\right. \\
+\mathrm{VE})\end{array}$ \\
\hline $\begin{array}{l}\text { Space } \\
\text { Complexity }\end{array}$ & $\mathrm{O}\left(\mathrm{V}^{\mathbf{2}}\right)$ & $\mathrm{O}\left(\mathrm{V}^{2}\right)$ & $\mathrm{O}\left(\mathrm{V}^{3}\right)$ & - \\
\hline $\begin{array}{l}\text { Negative } \\
\text { edges }\end{array}$ & No & Yes & Yes & Yes \\
\hline Single Source & Single & Single & Multiple & Multiple \\
All Sources & $\begin{array}{l}\text { Only one } \\
\text { source }\end{array}$ & $\begin{array}{l}\text { Only one } \\
\text { source }\end{array}$ & All sources & All sources \\
\hline
\end{tabular}




\section{CONCLUSIONS}

Since we have referenced some of the primary added substances of organizations and TCP/IP, you have the fundamental authentic past to check out the additional fundamental inconveniences of insurance in a united climate. Realizing how organizations are built offers you a higher data of what substantial or intelligent weaknesses are added through method of method for settling on one exact local area design over another. Knowing how bundles are molded offers you a higher data of ways they might be created or changed to acquire a specific reason. Knowing how bundles are sent and added offers you a higher data of what can appear to parcels as they visit from supply to objective. An accurate data of the basics of systems administration and TCP/IP is fundamental to distinguishing, data, and revising weaknesses to your merged climate.

\section{ACKNOWLEDGMENT}

I may first decide to convey my administrator, my PhD Guide, Dr. M. Robinson Joel, whose revel in changed into significant in forming the assessment questions and philosophy. Your keen remarks driven me to hone my scrutinizing and carried my artworks to a more elevated level. I may conjointly decide to convey my valuable pals for his valuable steerage over the span of my examinations. You provided me with the stuff that I needed to choose the right course and with accomplishment entire my composition. I may decide to famous my associates from my buddy Dr. D. Prasanna for his amazing joint effort manual for finish of my Research Paper. I need too many thank you to your impacted individual aide and for every one of the conceivable outcomes I was given to extra my assessment. Furthermore, I would decide to convey my people for his shrewd recommend and thoughtful ear. You're continually there for the benefit of me. These should be short and situated on the quit of the printed content sooner than the references.

\section{WEBSITE}

[1]. Packet routing abstract - Bing

[2]. How packet travels on network from source to destination - Algorithms and Me

[3]. Routing: How Do Packets Travel \& What

Happens inside a Router (fossbytes.com)

[4]. Packet flow in the same Network-

GeeksforGeeks

[5]. The Anatomy of a Packet Path (ICMP) from Source to Destination| The Anatomy of a Packet Path (ICMP) from Source to Destination | Pearson IT Certification

[6]. How packet travels on network from source to destination-Algorithms and Me

\section{REFERENCES}

1. Bar-Gera H (2002) Origin-based algorithm for the traffic assignment problem. Transportation Science, 36(4), 398-417. Chen, A., Jaya Krishnan, R. \& Tsai, W. K. (2002), "Faster FrankWolfe traffic assignment with new flow update scheme", Journal of Transportation Engineering, 128(1), 31-39. 
2. Dial RB (2006) A path-based user-equilibrium traffic assignment algorithm that obviates path storage and enumeration. Transportation Research Part B, 40B (10), 917-36.

3. Florian M, Constantin I, Florian D (2009) A new look at the projected gradient method for equilibrium assignment. Journal of the Transportation Research Board, Transportation Research Board of the National Academies, Washington DC, 2090, 10-16.

4. Peter Hofner and Bernhard Moller. Formal Aspects of Computing. 459-476.

5. Huang B, Wu Q, Zhan FB (2007) A novel heuristic for dynamic networks. International Journal of Geographical No. 6, 625-644, 2012. 